\title{
Muscle Functional Activation Patterns in Neck- Shoulder Muscles in Water and on Dry-Land with Resistance Training
}

\author{
Romualdo Castillo Lozano ${ }^{1 *}$ and Antonio Cuesta Vargas ${ }^{2,3}$ \\ ${ }^{1}$ Department of Physical Therapy, Spain \\ ${ }^{2}$ Faculty of Health Sciences, Spain \\ ${ }^{3}$ School Clinical Science, Australia \\ *Corresponding author: Romualdo Castillo Lozano, Lecturer, Department of Physical Therapy, Spain \\ Submission: 韭January 10, 2019; Published: 眥 February 12, 2019
}

\begin{abstract}
Background: Recent studies suggested that dipeptidyl peptidase-4 (DPP-4) inhibitors benefit to atherosclerosis-related cardiovascular diseases, but evidence was inconclusive. We aimed to determine the effects of dipeptidyl peptidase-4 inhibitors on Carotid Intima-Media Thickness in type 2 diabetes patients.

Methods: A literature search was conducted via electronic databases. The inclusion criteria were randomized controlled trials (RCTs) comparing DPP-4 inhibitors to placebo or anti-hyperglycaemic agent, study duration of no less than 12 weeks performed in type 2 diabetes patients. The primary outcome was the Intima-Media Thickness of Carotid. Random-effects model were applied to data synthesis.

Results: Five trials enrolling 762 and 761 patients for DPP-4 inhibitors and placebo or anti hyperglycaemic agent were included. The primary outcome of this meta-analysis was mean intima media thickness common carotid artery (mean-IMT-CCA) and left maximum intima media thickness of the common carotid artery (max-left-IMT-CCA). There were no obvious differences in mean-IMT-CCA (SMD: $-0.2466,95 \%$ CI [-0.6052, 0.1120], P=0.1777) over 12 months. And that's the same for 24 months (SMD: -0.5249, 95\%CI [-1.1492, 0.0994], $\mathrm{P}=0.0994$ ). There was no significant difference between DPP-4 Inhibitors and placebo or anti hyperglycemic agent in max-left-IMT-CCA, (SMD:0.2217, 95\%CI [-0.2602, 0.7035], P=0.3673). And remained same over 24 months (SMD:0.1064, 95\%CI [-0.3498, 0.5627], $\mathrm{P}=0.6475$ ).
\end{abstract}

Conclusion: There is no significant decrease on Carotid Intima-Media Thickness after DPP-4 inhibitors treatment over 12 and 24 weeks.

Keywords: Prevention and health promotion models; Childhood and adolescence

\section{Introduction}

Healthcare and fitness professionals must select exercises as a plan of care. Often the clinician struggles with which exercise to prescribe that best targets the shoulder muscles during functional movement patterns. For many years up to now, physiotherapy is a tool increasingly used for shoulder functional muscle reeducation procedures, being its aims to stabilize after normalization of structural joint dysfunction through the principles of proprioceptive neuromuscular facilitation and increased progressive resistance [1-3]. Improving arm active elevation commonly constitutes a large part of the muscle reeducation associated with many neck-shoulder conditions and is typically achieved with a combination of exercises active-assistive and active-resisted $[4,5]$.

Previous investigators reported shoulder exercises designed to explore not only the actual activity of the muscle but also the relative muscle activity ratio among synergist muscles [6-8]. Specifically, a high ratio of activation of any muscle related scapulohumeral rhythm was proposed as a contributing factor in abnormal scapular motion $[9,10]$. The excessive pectoralis major activation may lead to glenohumeral and scapulothoracic pathologies such as shoulder anterior joint translations or decreased compression forces on the glenoid [11-13]. When we study the kinematic of the shoulder we can see that sometimes there is an abnormal displacement of the humeral head during active elevation in participants with shoulder injury [14-16], although some shoulder injuries associated with normal movement and without pain, resulting from coupling forces from unaffected muscles that keep the relationship of the humeral head and glenoid fossa [17].

Glenohumeral instability is a multifaceted disorder with varying causes, degrees, and directions of instability. In normal shoulders the geometric center of the humeral head remained on the glenoid during scapular plane abduction [18]. The adaptation process of normal shoulder function with Aquatic Physiotherapy 
(AP) has been achieved through manipulation of the gestural speed, movement and the environment $[19,20]$. The aquatic environment showed advantages based on several important bioengineering properties: basic forces, buoyancy, drag, inertial forces, hydrostatic pressure and specific heat. It is interesting to understand the force vectors acting on an arm movement with resistance training in the water and continuously moving the metacenter (alignment-balance arm) [21,22]; and progressive gain autonomy in the water that lead to therapeutic advantages for to readapt the shoulder function and turn it into a functional structure $[18,21,23]$.

Common isotonic muscle reeducation exercises for the rotator cuff and deltoid muscles, whether for impingement or instability, involve lifting a weighted object in the scapular plane [15]. In a study, surface electromyography (SEMG) activity during caption with humeral external rotation with light weights peaked during the $120^{\circ}$ to $150^{\circ}$ arc of elevation. However, patients with rotator cuff tendinitis may not be able to reach this position. Therefore, it would be advantageous to identify and introduce a variable to shift the peak activity into a lower, safer range of motion. Working on the aquatic environment can be combined with the dry-land as therapeutic goals. There is a protocol for muscle reeducation of the shoulder on dry-land and in water $[20,21]$, but it has not established yet a protocol through resistance training for restoration of normal biomechanics of the shoulder. This model approach in the muscle reeducation process of the shoulder with resistance training lacks a scientific justification that demonstrates the validity and effectiveness as well as the methodological progression to follow. This conflict can confuse the process of muscle reeducation for the shoulder girdle [5].

To provide the necessary evidence in the process of muscle reeducation of shoulder injuries more research is required. Therefore, we use the SEMG to examine the level of muscle activation $[4,17,19]$. Presently, no one has provided normalized muscle activation ratios for the trunk or back muscles during the performance of scapular plane abduction, caption or flexion in standing against resistance provided by dumbbell free weights in water vs on dry-land. The effectiveness of strengthening shoulder muscles has been demonstrated using a series of exercises performed primarily with the arm below $45^{\circ}$ of shoulder elevation. However, specific exercises have been identified that facilitate high SEMG activity of the rotator cuff and scapular musculature that require humeral motions at or above $45^{\circ}$ of shoulder elevation $[24,25]$. The identified exercises not only elicit high levels of muscular activity in healthy individuals but also simulate functional activities. Physical therapy supplemented with SEMG form a perfect tool for shoulder muscle reeducation both in-water and on dry-land by adding a resistance training in water (inflatable float arm) and dry-land (1kg dumbbell) (Figure 1).
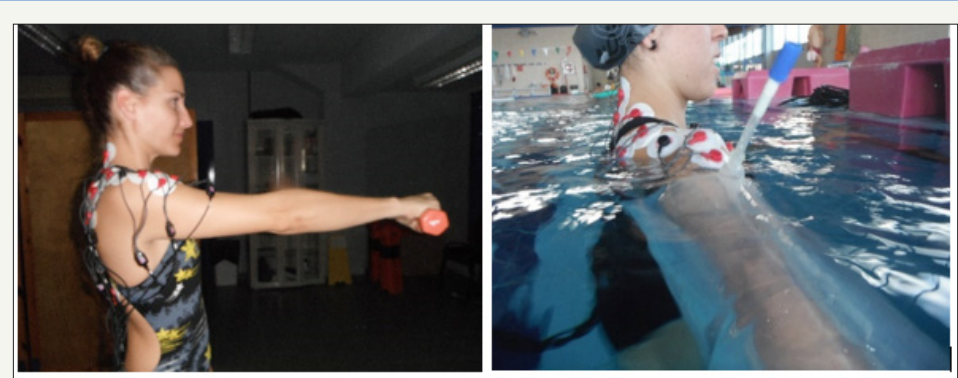

Figure 1: Execution of the movements on dry-land (1 kg dumbbell) and in water (inflatable float arm).

The purpose of this study is to compare shoulder muscle activation during arm elevation with resistance training through the different movement planes at different during in-water and dry-land exercise.

\section{Methods}

\section{Participants}

We studied the musculature of the right shoulder of 16 healthy participants ( 8 males and 8 females). Before collecting any data, Participants voluntarily signed an informed consent previously approved for this study. The mean age of participants was 26 years with a standard deviation of 4.48, ranging from 20 to 34 years. Most participants in this study also reported a history of upper-body weight training. This study was previously approved by Research Ethics Committee of the Faculty of Health Sciences from the University of Malaga. After, they were measured for anthropometric criteria according to the International Society for the Advancement of Kin anthropometry [14].

\section{Procedures}

Bipolar disc adhesive surface electrodes (Ambu A/S, Neuroline, $72001 \mathrm{k}$, Ballerup, Denmark) measuring 3 to $5 \mathrm{~mm}$ in surface diameter were placed $1-3 \mathrm{~cm}$ apart (motor point) for each muscle in accordance with the methods described by Surface Electromyography for the Non-Invasive Assessment of Muscles [26]. Adhesive surface electrodes were used on all participants after skin cleaning, application of an adhesive spray (Tenos Spray $^{\mathrm{TM}}$ ) and protection by a waterproof adhesive tape (Tegaderm, 3M, St Paul, US). Activity was analyzed for the cervical erector spinae, trapezius, supraspinatus, infraspinatus, anterior deltoid and middle pectoralis major (sternal fibers) and latissimus dorsi. All electrodes were connected to a console SEMG ME6000 protected of the water with a cover for this use; and the signal recorded with Mega Win 3.0.1 software. Sampling was performed at $1000 \mathrm{H}_{\mathrm{z}}$ and root mean squared averaging applied to obtain data collection.

After collecting the raw data, it was fed into the software where a low pass filter (Butterworth, $4^{\text {th }}$ order, $10-400 \mathrm{H}_{\mathrm{z}}$ ) was applied to 
eliminate noise pollution, with a bandwidth of $20 \mathrm{H}_{z^{\prime}}$, an attenuation of $60 \mathrm{~dB}$ and a maximum frequency of $400 \mathrm{H}_{\mathrm{z}}$. Maximum voluntary isometric contraction (MVC) tests were performed for further normalization of the SEMG signal [19,27-29]. According to the randomization (dry-land or water), participants performed three movements (flexion, abduction and scaption through a range of 0 $\left.{ }^{\circ}-90^{\circ}\right)$ at three movement speeds $\left(30 \% \mathrm{sec}, 45^{\circ} / \mathrm{sec}\right.$ and $90 \%$ sec that were determined by a metronome) on the environment assigned. The possibilities were: water or dry-land. The intervention on dryland tests was performed in a room (ambient air-temperature of $22.0{ }^{\circ} \mathrm{C}$ ) and supplemented all movements and speeds with a $1 \mathrm{~kg}$ dumbbell that the participant has in his hand. The in-water tests were performed in an indoor pool (depth $1.30 \mathrm{~m}$, water at $31.0^{\circ} \mathrm{C}$ and pool ambient air-temperature at $32.8{ }^{\circ} \mathrm{C}$ ). Participants were immersed to the outer-edge of the acromion with an inflatable float arm of $30 \mathrm{~cm}$ long and $15 \mathrm{~cm}$ wide (from mid arm to mid-forearm). In both environments, the participants were in standing.

Following electrode attachment, each participant was given an explanation about the execution speed (neutral humeral rotation). Testing commenced once the participant fully understood the procedure and the execution was performed correctly. All movements, speeds and times were controlled by the same researcher. Five repetitions were collected for each movement and speed, both dry-land and in-water for subsequent analysis (average of 5 values).

\section{Statistical Analyses}

The analysis was performed with SPSS version 15 for Windows. The dependent variables were normalized root-mean-square values obtained for each muscle in participants. We use the average of the five repetitions. The Kolgomorov-Smirnov test was used as determined by the variable's normality of distribution. The means difference was determined with Wilcoxon or Student-t test paired according to normality; the effect size with Cohen's $d$ and the analysis of reliability of measurements was performed using the Intraclass Correlation Coefficient. The statistical significance level was set at $\mathrm{p}<05$.

\section{Result}

Table 1: Descriptive statistics of the sample.

\begin{tabular}{|c|c|c|}
\hline & Mean & Stand Dev. \\
\hline Age (years) & 26.06 & 4.47 \\
\hline Weight (kg) & 66.34 & 10.19 \\
\hline Size (m) & 1.7 & 0.095 \\
\hline Bistyloid Diameter (mm) & 50.37 & 3.42 \\
\hline Arm land weight (kg) & 3.32 & 0.5 \\
\hline Arm water weight (kg) & 0.57 & 0.03 \\
\hline Arm fat weight (kg) & 0.45 & 0.01 \\
\hline Arm bone weight (kg) & 0.28 & 0.01 \\
\hline Arm muscle weight (kg) & 2.59 & 0.39 \\
\hline Distance - acromion-to wrist (cm) & 52.75 & 12.6 \\
\hline Biceps skinfold (mm) & 7.75 & 6.94 \\
\hline Triceps skinfold (mm) & 14.31 & 8.08 \\
\hline Subscapular skinfold (mm) & 12.06 & 6.55 \\
\hline Percentage fat & 13.55 & 9.43 \\
\hline
\end{tabular}

Table 2: Descriptive statistics of the movement on dry-land vs in water.

\begin{tabular}{|c|c|c|c|c|c|c|c|c|c|}
\hline LAND & $\begin{array}{l}\text { ABD } 90^{\circ} \% \\
\text { seg }\end{array}$ & ABD $45 \% /$ seg & $\begin{array}{l}\mathrm{ABD} 30 \% \\
\text { seg }\end{array}$ & SC $90 \% / \mathrm{seg}$ & $\begin{array}{l}\text { SC } 45^{\circ} \% \\
\text { seg }\end{array}$ & $\begin{array}{l}\text { SC } 30 \% \\
\text { seg }\end{array}$ & $\begin{array}{l}\text { FL } 90 \% \\
\text { seg }\end{array}$ & $\begin{array}{l}\text { FL } 45 \% \\
\quad \text { seg }\end{array}$ & $\begin{array}{c}\text { FL } 30 \% \\
\text { seg }\end{array}$ \\
\hline Mean Gesture $(\mu \mathrm{V})$ & 376.35 & 427.74 & 421.39 & 360.33 & 368.87 & 321.14 & 360.22 & 367.78 & 315.53 \\
\hline Standard Deviation & \pm 284.10 & \pm 340.63 & \pm 313.13 & \pm 308.60 & \pm 330.15 & \pm 255.72 & \pm 297.48 & \pm 342.21 & \pm 228.64 \\
\hline WATER & $\begin{array}{l}\mathrm{ABD} 90^{\circ} \% \\
\text { seg }\end{array}$ & ABD $45 \% /$ seg & $\begin{array}{l}\mathrm{ABD} 30 \% \\
\quad \operatorname{seg}\end{array}$ & SC $90 \% /$ seg & $\begin{array}{l}\text { SC } 45^{\circ} \% \\
\text { seg }\end{array}$ & $\begin{array}{c}\text { SC } 30 \% \\
\text { seg }\end{array}$ & $\begin{array}{l}\text { FL } 90^{\circ} \% \\
\text { seg }\end{array}$ & $\begin{array}{l}\text { FL } 45 \% \\
\text { seg }\end{array}$ & $\begin{array}{c}\text { FL } 30 \% \\
\text { seg }\end{array}$ \\
\hline Mean Gesture $(\mu \mathrm{V})$ & 535.34 & 565.85 & 521.6 & 545.8 & 553.4 & 481.48 & 445.84 & 503.2 & 527.51 \\
\hline Standard Deviation & \pm 530.67 & \pm 502.47 & \pm 504.78 & \pm 473.46 & \pm 505.19 & \pm 381.65 & \pm 442.28 & \pm 416.27 & \pm 515.75 \\
\hline $\begin{array}{c}\text { Intra-Class Correla- } \\
\text { tions }\end{array}$ & 0.445 & 0.413 & 0.378 & 0.511 & 0.552 & 0.307 & 0.324 & 0.235 & 0.504 \\
\hline Cohen's d & -0.4 & -0.32 & -0.24 & -0.46 & -0.43 & -0.5 & -0.23 & -0.36 & -0.53 \\
\hline Effect-Size $r$ & -0.2 & -0.16 & -0.12 & -0.23 & -0.21 & -0.24 & -0.11 & -0.17 & -0.26 \\
\hline
\end{tabular}

ABD: Abduction; SC: Scaption; FL: Flexion. 
Here, we show the anthropometric characteristics and the descriptive statistics of the participants in this study (Table 1). The values of muscle activity during arm elevation with resistance training in the three gestures and three speeds are presented in (Table 2).

Later, the values of muscle activity were normalized to MVC (Table 3). To classify and compare the relative SEMG activation of each muscle, we used a system which classified muscular activation into 4 categories:
A. $\operatorname{Low}(<20 \%$ MVC $)$

B. Moderate $(20-40 \%$ MVC)

C. High (41-60\% MVC)

D. Very high $(>60 \%$ MVC)

The proposed scheme agreed with subsequent researchers who proposed that the threshold value for muscle strength gains during exercise requires SEMG activation greater than $50-60 \%$ [30]; (Table 3).

Table 3: Muscles and speeds with significant differences during movements on dry-land and water environments.

\begin{tabular}{|c|c|c|c|c|c|c|c|c|c|c|}
\hline & \multicolumn{4}{|c|}{ FLEXION } & \multicolumn{3}{|c|}{ ABDUCTION } & \multicolumn{3}{|c|}{ SCAPTION } \\
\hline & $90^{\circ}{ }_{S}$ SUPR & $\begin{array}{l}90^{\circ} \\
\text { PECT }\end{array}$ & $45^{\circ}{ }_{\text {_PECT }}$ & $\begin{array}{c}30^{\circ} \\
\text { PECT }\end{array}$ & $\begin{array}{c}30^{\circ} \\
\text { SUPR }\end{array}$ & $\begin{array}{c}90^{\circ} \\
\text { PECT }\end{array}$ & $\begin{array}{l}30^{\circ} \\
\text { PECT }\end{array}$ & $\begin{array}{c}30^{\circ} \\
\text { SUPR }\end{array}$ & $\begin{array}{c}90^{\circ} \\
\text { PECT }\end{array}$ & $\begin{array}{c}30^{\circ} \\
\text { DELTM }\end{array}$ \\
\hline Land Mean (\%) & 50.9 (III) & 59.4 (III) & 42.4 (III) & 56.6 (III) & 90.2 (IV) & $10.6(\mathrm{I})$ & 23.1 (II) & 35.7 (II) & 26.9 (II) & 62.1 (IV) \\
\hline Water Mean (\%) & $19.3(\mathrm{I})$ & 97.2 (IV) & 80.2 (IV) & 79.6 (IV) & 43.7 (III) & 79.8 (IV) & 97.5 (IV) & 23.8 (II) & 90.4 (IV) & 72.5 (IV) \\
\hline Sig. & 0.026 & 0.039 & 0.049 & 0.05 & 0.05 & 0.044 & 0.034 & 0.05 & 0.015 & 0.049 \\
\hline
\end{tabular}

$90^{\circ}$ _SUPR: Standardization Land vs Water at $90^{\circ} / \mathrm{sec}$ of the Supraspinatus muscle; $90^{\circ}$ _PECT: Standardization Land vs Water at $90^{\circ} / \mathrm{sec}$ of the Pectoralis muscle; $45^{\circ}$ _PECT: Standardization Land vs Water at $45^{\circ} / \mathrm{sec}$ of the Pectoralis

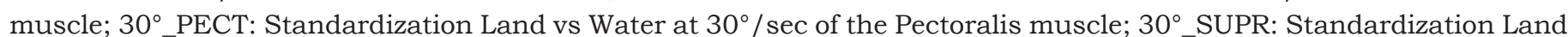
vs Water at $30^{\circ} / \mathrm{sec}$ of the Supraspinatus muscle, $30^{\circ}$ _DELTM: Standardization Land vs Water at $30^{\circ} /$ sec of the Deltoid Middle muscle.

\section{Discussion}

To our knowledge, no previous research has normalized SEMG activity of the 8 muscles of shoulder-neck for caption, abduction and flexion with resistance training in water/on dry-land. Additionally, a limited amount of research exists reporting the normalized SEMG values of the shoulder and scapular muscles for these movements. The primary purpose of this cross-sectional study was to determine if the muscular activation shoulder-neck with resistance training in two different environments can be effective to create a muscle reeducation procedure. In this study, there weren't statistically significant differences according to the randomization of the environment. The normalized SEMG values were chosen to discuss the relative muscle activation. SEMG analysis does not genuinely provide a direct measure of muscle strength; instead, it merely measures the neuromuscular activity underneath the surface electrode.

However, normalized muscle recruitment can be used to determine which exercise pattern produces the highest external demand upon a muscle. In this study, we observed changes in muscle activation when we compared the performance in both environments $(p<0.05)$. When we place resistance training in different environments, we found that muscle activation in the execution on dry-land with a $1 \mathrm{~kg}$ dumbbell is lower in all measured muscles, movements and speeds; regarding the execution in water with an inflatable float arm (Table 2).

\section{Activation in each of the gestures}

We note that activation percentage in abduction with resistance training gesture is lower in the three speeds on dry-land with an average of 408 microvolts versus 550 microvolts in water, with the small difference that the supraspinatus $(\mathrm{p}=.05)$ at slow speed $(30 \%$ sec) presents greater activation on dry-land relative in water [15]. In this movement, the pectoralis major shows greater activation at low/high speed $(\mathrm{p}=.034, \mathrm{p}=.044)$ in water compared to dry-land [11-13]. Caption. We see that there is more variability on dry-land/ in water since it is a gesture combined the abduction and flexion. The average activation on dry-land is 349 microvolts versus 526 microvolts in water. In this gesture, the supraspinatus $(\mathrm{p}=.05)$ also shows greater activation on dry-land against water at low speeds.

However, there are also differences in the pectoralis major $(p=.015)$ at high speeds as the main stabilizer and the middle deltoid muscle $(\mathrm{p}=.049)$ in water at low speeds. During scaption, the arms were positioned in the scapular plane (i.e., $30^{\circ}$ anterior to the frontal plane) activating the shoulder horizontal abductor including supraspinatus and infraspinatus [15,31] and thus inhibiting pectoralis major activation also reported that greater demand during weight bearing exercises increases pectoralis major activation [32]. Flexion: The muscle activation for the three speeds on dry-land present an average of 347 microvolts versus 491 microvolts in water. The supraspinatus $(\mathrm{p}=.026)$ has a higher activation on dry-land regarding in water, but this time at high 
speeds as acts stabilizer of the glenohumeral joint and no main executor. However, in water the pectoralis major becomes more important in the movement at all speeds ( $\mathrm{p}=.05 ; \mathrm{p}=.49 ; \mathrm{p}=.039)$ $[11,12,32]$.

\section{Muscular working-load in each speed}

In this study it was demonstrated that when movement is performed at higher speed $(90 \% \mathrm{sec})$ with resistance training seems to be the point where the water resistance form with an inflatable float arm is greater than the gravity column with the $1 \mathrm{~kg}$ dumbbell on dry-land. This speed indicated greater contractility in the pectoralis major at all movements in water respect on dryland; and less contractility in the supraspinatus during flexion in water compared on dry-land. These findings indicated that at this movement a high speed, the buoyancy is overcome in the waters for the isokinetic resistance for pectoralis major muscle. Note that at this speed, the supraspinatus has a lower activation in water compared on dry-land. This can be explained as the supraspinatus intervenes in the first $30^{\circ}$ degrees of motion and those grades with resistance training are made very assisted.

This finding can be explained by the reciprocal inhibition mechanism, that it is due to afferent impulses from agonist muscle spindles stimulating an inhibitory interneuron in the spinal cord, which causes inhibition of the alpha motor neuron to the antagonist muscle [33]. This leads to the assumption that there is an interface where transition occurs from resisted (pectoralis major) movement to that of active-assisted (supraspinatus) with in the aquatic environment $[11,31]$. In medium speed $(45 \% \mathrm{sec})$ with resistance training there is a mismatch and connection between movements on dry-land and in water. The only muscle that shows statistically significant changes is the pectoralis major in moving flexion. The buoyancy (inflatable float arm) is overcome by eccentric activation to slow motion in water and the gravity overcome by the $1 \mathrm{~kg}$ dumbbell.

Consequently, there is an increase in contractility during the medium velocity in-water movement resulting in a significant increase in relative muscle activation in the pectoralis major. This significant increase in muscle activation in-water indicated that medium speed movement in-water provided greater eccentric activation helping by an inflatable float arm [18]. The mean muscle activation in the movements of low speed $(30 \% \mathrm{sec})$ in the supraspinatus muscle during abduction and scaption is less in the aquatic environment while the pectoralis major during abduction and flexion; and the middle deltoid during scaption are activated more in water environment. The decrease of in-water speed resulted in a decrease in activation of the supraspinatus muscle [34], because at low speed the water assists the movement instead of resisting or braking. However, for the middle deltoid and pectoralis major are not met this assumption since they are essential muscles for movement at low speeds with inflatable float arm. Again, from our study the importance of the pectoralis major, middle deltoid and supraspinatus in the control of movement execution is demonstrated with resistance training.

\section{Clinical implications}

Glenohumeral instability and the presence of atrophy muscles of the shoulder are a relatively common condition affecting $2 \%$ of the general population $[15,16]$. In-water with an inflatable float arm the middle deltoid and pectoralis major are primarily responsible for giving stability to the shoulder [35]. This is likely achieved through a coupled action where pectoralis major is working eccentrically and consequently providing a relative inferior translation to that of the superior translation of the deltoid, because the shoulder makes some rotational adjustments to improve functional capacity in performing an activation open kinetic chain segment (proximal to distal). Consequently, a net stabilization results analogous to that achieved by the coupled supraspinatus in the dry-land setting, inhibited in water when applying an inflatable float arm in low speeds [36].

In this study, the variables that could explain the creation of a new model for AP are speed, the progression of work in different environments and the application of active resistance training in the ideal environment. Without proper progression and order of execution in the process of muscle muscle reduction, the shoulder injury can worsen [21]. The beginning would be held on dry-land at all speeds and movements with the $1 \mathrm{~kg}$ dumbbell (analytical work). This will allow us to step into the water with an inflatable float arm (synergistic work). This progression on dry-land/in water gives us greater security in the standardization process of the shoulder injuries: first stimulating abduction (57,48\%); after flexion $(60.72 \%)$ and finally scaption (61.91\%).

\section{Limitations and Strengths of the Study}

In this study we found some limitations: A relatively small sample size (16 participants); muscle crosstalk (potential confounding influence for SEMG) and accuracy of skin surface electrode location over the muscle belly throughout dynamic muscle contractions. Additionally, the external validity of our study is limited because the results only apply to young, healthy individuals and not necessarily to the general population or others with shoulder pathology. However, this study provides a method for continuing the muscle reeducation process after the application of the procedures described by Kelly et al. [19] and Castillo-Lozano et al. [20] in their respective studies, adjusting the muscle reeducation with resistance training.

\section{Conclusion}

For muscle reeducation process aiming for active shoulder movement, activity should first be performed on dry-land with $1 \mathrm{~kg}$ dumbbell at all speeds, as the muscle activation is lower than in water. Finally, we will make the same performance in water with an inflatable float arm. The water form resistance and the retention aid an inflatable float arm, cause activation in water is greater than on dry-land. For muscle reeducation, the pectoral muscle has a very important role in the stabilization of the injury shoulder at all speeds and movement in-water with little influence from the latissimus dorsi and cervical erector spinae muscles. Progression 
in the movements in each environment will be introduction of abduction, after flexion and finally scaption. Execution speed will progressively slower to faster. The setting of the intervention protocol, choice of movement type, speed and environment are the main variables that must be controlled in the process of functional muscle reeducation of the shoulder by AP.

\section{Ethical Approval}

This study was previously approved by Research Ethics Committee of the Faculty of Health Sciences from the University of Malaga.

\section{Acknowledgement}

The authors wish to thank Patronato Municipal de Torremolinos (Malaga) for access to its facilities and his generous cooperation during the project. No funding was sought or received for the completion of this research project.

\section{References}

1. Boyle AM (1981) The bad ragaz ring method. Physiotherapy 67(9): 265268.

2. Campion MR (1990) Adult hydrotherapy: A practical approach Heinemann Medical Books, Oxford, USA

3. Martin J (1981) The halliwick method. Physiotherapy 67(10): 288-291.

4. Fujisawa H, Suenaga N, Minami A (1998) Electromyographic study during isometric exercise of the shoulder in head-out water immersion. J Shoulder Elbow Surg 7(5): 491-494.

5. Hawkes DH, Alizadehkhaiyat O, Kemp GJ, Fisher AC, Margaret M, et al. (2012) Shoulder muscle activation and coordination in patients witha massive rotator cuff tear: An electromyographic study.J Orthop Research 30(7): 1140-1146.

6. Cools AM, Dewitte V, Lanszweert F, Notebaert D, Roets A, et al. (2007) Rehabilitation of scapular muscle balance: which exercises to prescribe? Am J Sports Med 35(10): 1744-1751.

7. Cools AM, Dewitte V, Lanszweert F, Notebaert D, Roets A, et al. (2007) Rehabilitation of scapular muscle balance: which exercises to prescribe? Am J Sports Med 35(10): 1744-1751.

8. Ludewig PM, Hoff MS, Osowski EE, Meschke SA, Rundquist PJ (2004) Relative balance of serratus anterior and upper trapezius muscle activity during push-up exercises. Am J Sports Med 32(2): 484-493.

9. Martins J, Tucci HT, Andrade R, Araújo RC, Bevilaqua GD, et al. (2008) Electromyographic amplitude ratio of serratus anterior and upper trapezius muscles during modified push-ups and bench press exercises. J Strength Cond Res 22(2): 477-484.

10. Hiengkaew V, Wichaiwong K, Chaiyakul S, Deesin A (2003) Concerning the pectoralis major in active reaching exercise. Electromyogr Clin Neurophysiol 43(3): 157-163.

11. Kim B, Gong W, Lee S (2010) The effect of push-up plus exercise with visual bio feedback on the activity of shoulder stabilizer muscles for winged scapula. J Phys Ther Sci 22(4):355-358.

12. Konrad GG, Jolly JT, Labriola JE, McMahon PJ, Debski RE (2006) Thoracohumeral muscle activity alters glenohumeral joint biomechanics during active abduction. J Orthop Res 24(4): 748-756.

13. Labriola JE, Lee TQ Debski RE, McMahon PJ (2005) Stability and instability of the glenohumeral joint: the role of shoulder muscles. Shoulder Elbow Surg 14(Suppl 1): 32S-38S.
14. McMahon PJ, Chow S, Sciaroni L, Yang BY, Lee TQ (2003) A novel cadaveric model for anterior-inferior shoulder dislocation using forcible apprehension positioning. J Rehabil Res Dev 40(4): 349-359.

15. Marfell JM, Olds T, Stewart A, Carter L (2006) International standards for anthropometric assessment. Potchefstroom, South Africa.

16. Terrier A, Reist A, Vogel A, Farron A (2007) Effect of supraspinatus deficiency on humerus translation and glenohumeral contact force during abduction. Clin Biomech (Bristol, Avon) 22(6): 645-651.

17. Teyhen DS, Miller JM, Middag TR, Kane EJ (2008) Rotator cuff fatigue and glenohumeral kinematics in participants without shoulder dysfunction. J Athl Train 43(4): 352-358.

18. Yamaguchi K, Sher JS, Andersen WK, Garretson R, Uribe JW, et al. (2000) Glenohumeral motion in patients with rotator cuff tears: a comparison of asymptomatic and symptomatic shoulders. J Shoulder Elbow Surg 9(1): 6-11.

19. Kelly BT, Kadrmas WR, Kirkendall DT, Speer KP (1996) Optimal normalization tests for shoulder muscle activation: an EMG study. J Orthop Res 14(4): 647-653.

20. Castillo LR, Cuesta VA, Gabel CP (2013) Analysis of arm elevation muscle activity through different movement planes and speeds during in-water and dry-land exercise. J Shoulder Elbow Surg 23(2): 159-165.

21. Kelly BT, Roskin LA, Kirkendall DT, Speer KP (2000) Shoulder muscle activation during aquatic and dry land exercises in nonimpaired participants. J Orthop Sports Phys Ther 30(4): 204-2 10.

22. Castillo LR, Cuesta VAI (2013) A comparison land-water environment of maximal voluntary isometric contraction during manual muscle testing through surface electromyography. BMC Sports Sci Med Rehabil 5(1): 28.

23. Vorontsov AR, Rumyantsev VA (2000) Resistive forces in swimming. In: Zatsiorsky VM (ed.), Biomechanics in Sport International Olympic Committee, Oxford, USA, pp. 184-204

24. Jerosch J, Wüstner P (2002) Effect of a sensorimotor training program on patients with subacromial pain syndrome. Unfallchirurg, pp. 36-43.

25. Hardwick DH, Beebe JA, McDonnell MK, C Lang (2006) A comparison of serratus anterior muscle activation during a wall slide exercise and other traditional exercises. J Orthop Sports Phys Ther 36(12): 903-910.

26. Reinold MM, Wilk KE, Fleisig GS (2004) Electromyographic analysis of the rotator cuff and deltoid musculature during common shoulder external rotation exercises. Journal of Orthopaedic and Sports Physical Therapy 34(7): 385-394.

27. Hermens H, Freriks BS (1997) The state of the art on sensors and sensor placement procedures for surface electromyography, a proposal for sensor placement procedures. Deliverable of the SENIAM Project, Rousing Research and Development, USA.

28. Alberton CL, Cadore EL, Pinto SS, Tartaruga MP, Silva EM, et al. (2011) Cardiorespiratory, neuromuscular and kinematic responses to stationary running performed in water and on dry land. European Journal Applied Physiology 111(6): 1157-1166.

29. Hislop HJ, Montgomery J (2007) Muscle testing: Techniques of manual examination. St. Louis, USA

30. Silvers W, Dolny D (2010) Comparison and reproducibility of sEMG during manual muscle testing on land and in-water. J Electro myogrKinesiol 21(1): 95-101.

31. Youdas JW, Arend DB, Exstrom JM, Helmus TJ, Rozeboom JD, et al. (2012) Comparison of muscle activation levels during arm abduction in the plane of the scapula vs. proprioceptive neuromuscular facilitation upper extremity patterns. J Strength Cond Res 26(4): 1058-1065.

32. Neumann DA (2010) Kinesiology of the musculoskeletal system: Foundations for muscle reeducation ( $2^{\text {nd }}$ edn), Mosby, USA. 
33. Uhl TL, Carver TJ, Mattacola CG, Mair SD, Nitz AJ (2003) Shoulder musculature activation during upper extremity weight-bearing exercise. J Orthop Sports Phys Ther 33(3): 109-117.

34. Stuart I (2007) Human physiology: mechanism of contraction and neural control. (10 ${ }^{\text {th }}$ edn), McGraw-Hill, New York, USA.
35. Kelly BT, Cooper LW, Kirkendall DT, Speer KP (1997) Technical considerations for EMG research on the shoulder. Clinical Orthopaedics and Related Research 335(335): 140-151.

36. Ikuta Y, Matsuda Y, Yamada Y, Kida N, Oda S, et al. (2012) Relationship between decreased swimming velocity and muscle activity during $200 \mathrm{~m}$ front crawl. Eur J Appl Physiol 112(9): 3417-3429. International License

For possible submissions Click Here

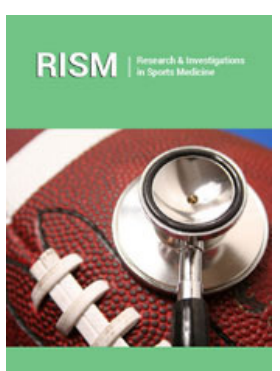

Research \& Investigations in Sports Medicine

\section{Benefits of Publishing with us}

- High-level peer review and editorial services

- Freely accessible online immediately upon publication

- Authors retain the copyright to their work

- Licensing it under a Creative Commons license

- Visibility through different online platforms 